\title{
Acupuncture decreased the risk of coronary heart disease in patients with rheumatoid arthritis in Taiwan: a Nationwide propensity score-matched study
}

Mei-Yao Wu', Ming-Cheng Huang ${ }^{1,2}$, Hou-Hsun Liao ${ }^{2,3}$, Jen-Huai Chiang ${ }^{4,5}$, Yu-Chen Lee ${ }^{1,6}$, Chung-Y Hsu', Mao-Feng Sun ${ }^{1,2^{*+}}$ and Hung-Rong Yen ${ }^{1,2,8,9,10,11^{*+}}$ (D)

\begin{abstract}
Background: Patients with rheumatoid arthritis (RA) have a higher risk of coronary heart disease (CHD). Acupuncture, a commonly used treatment for patients with RA, has not been reported to prevent CHD in patients with RA. We aimed to assess the risk of developing CHD in acupuncture users and non-users of patients with RA.

Methods: We identified 29,741 patients with newly diagnosed RA from January 1997 to December 2010 from the Registry of Catastrophic IIIness Patients Database from the Taiwanese National Health Insurance Research Database. Among them, 10,199 patients received acupuncture (acupuncture users), and 19,542 patients did not receive acupuncture (noacupuncture users). After performing 1:1 propensity score matching by sex, age, baseline comorbidity, conventional treatment, initial diagnostic year, and index year, there were 9932 patients in both the acupuncture and no-acupuncture cohorts. The main outcome was the diagnosis of CHD in patients with RA in the acupuncture and no-acupuncture cohorts.

Results: Acupuncture users had a lower incidence of $\mathrm{CHD}$ than non-users (adjusted $\mathrm{HR}=0.60,95 \% \mathrm{Cl}=0.55-0.65$ ). The estimated cumulative incidence of CHD was significantly lower in the acupuncture cohort (log-rank test, $p<.001$ ). Subgroup analysis showed that patients receiving manual acupuncture of traditional Chinese medicine style, electroacupuncture, or combination of both all had a lower incidence of CHD than patients never receiving acupuncture treatment. The beneficial effect of acupuncture on preventing CHD was independent of age, sex, diabetes mellitus, hypertension, hyperlipidemia, and statins use.
\end{abstract}

Conclusions: This is the first large-scale study to reveal that acupuncture might have beneficial effect on reducing the risk of CHD in patients with RA. This study may provide useful information for clinical utilization and future studies.

Keywords: Acupuncture, Coronary heart disease, National Health Insurance Research Database, Rheumatoid arthritis, Taiwan

\section{Background}

Rheumatoid arthritis (RA) is one of the most prevalent chronic and systemic inflammatory disorders, with the characteristics of joint destruction, functional disability, and early death [1]. Autoantibody production, synovial inflammation, cartilage and bone destruction are the main pathological findings in RA patients [2]. Current

\footnotetext{
* Correspondence: maofeng@mail.cmuh.org.tw; hungrongyen@gmail.com ${ }^{\dagger}$ Mao-Feng Sun and Hung-Rong Yen contributed equally to this work. ${ }^{1}$ Department of Chinese Medicine, China Medical University Hospital, 2 Yude Road, North District, Taichung 404, Taiwan

Full list of author information is available at the end of the article
}

pharmacotherapies of RA include non-steroidal anti-inflammatory drugs (NSAIDs), glucocorticoids, and disease-modifying anti-rheumatic drugs (DMARDs) [3]. DMARDs, including synthetic and biological DMARDs, have been demonstrated to suppress inflammation to reduce structural damage.

The high mortality rate of patients with RA was mainly because of the significantly higher risk of cardiovascular diseases in patients with RA than in the general population [4]. The previous study revealed that patients with RA had an approximately twofold higher risk of coronary heart disease (CHD) [5]. The risk of CHD in patients with RA is

(c) The Author(s). 2018 Open Access This article is distributed under the terms of the Creative Commons Attribution 4.0 International License (http://creativecommons.org/licenses/by/4.0/), which permits unrestricted use, distribution, and 
highly associated with the traditional risk factors of CHD, which include hypertension, diabetes mellitus (DM), hyperlipidemia, smoking, obesity, and physical inactivity [6]. Chronic inflammation and immune dysregulation play an important role in inducing atherosclerosis and endothelial dysfunction in patients with RA [7]. Among current pharmacotherapy of RA, methotrexate (MTX) might prevent ischemic cardiovascular disorders in patients with RA [8]. Etanercept, a tumor necrosis factor- $\alpha$ (TNF- $\alpha$ ) inhibitor, was demonstrated to attenuate endothelial dysfunction in a rat model [9]. These findings suggest that anti-inflammatory therapy may reduce the CHD risk in patients with RA.

A previous cohort study revealed that approximately $27.3 \%$ of patients with RA were traditional Chinese medicine (TCM) users in Taiwan, and approximately $23.6 \%$ of TCM users with RA received acupuncture [10]. The anti-inflammatory mechanisms of acupuncture have been demonstrated in murine models [11, 12]. Previous clinical trials have demonstrated that acupuncture suppressed inflammation in patients with RA [13, 14]. However, no prior study evaluated whether acupuncture can prevent $\mathrm{CHD}$ in patients with RA. To investigate whether acupuncture decreases the incidence of $\mathrm{CHD}$ in patients with RA, we conducted this cohort study.

\section{Methods}

\section{Data sources}

The Taiwanese National Health Insurance Research Database (NHIRD) is a good database for the cohort study because it contains the long-term follow-up data of most of the population in Taiwan. The National Health Insurance (NHI) program was implemented in 1995 in Taiwan by the National Health Insurance Administration. TCM services have been reimbursed through the NHI since 1996 [15]. The reimbursed population reached more than $99.5 \%$ in 2015 [16]. NHIRD is a big database that can prevent sampling bias [17].

The data source of this study was the Registry of Catastrophic Illness Patients Database; patients with autoimmune diseases, such as RA [18], systemic lupus erythematosus, multiple sclerosis, Sjögren syndrome, and cancer, are included in this database, which is a subsection of the NHIRD [19]. All of the patients with RA in the database are given catastrophic illness certificates based on the clinical and laboratory diagnoses by rheumatologists. The NHI program waived RA patients' copayments for receipt of RA-related treatments, including Western medicine and TCM. In this database, the International Classification of Diseases, Ninth Revision, Clinical Modification (ICD-9-CM) codes were the most

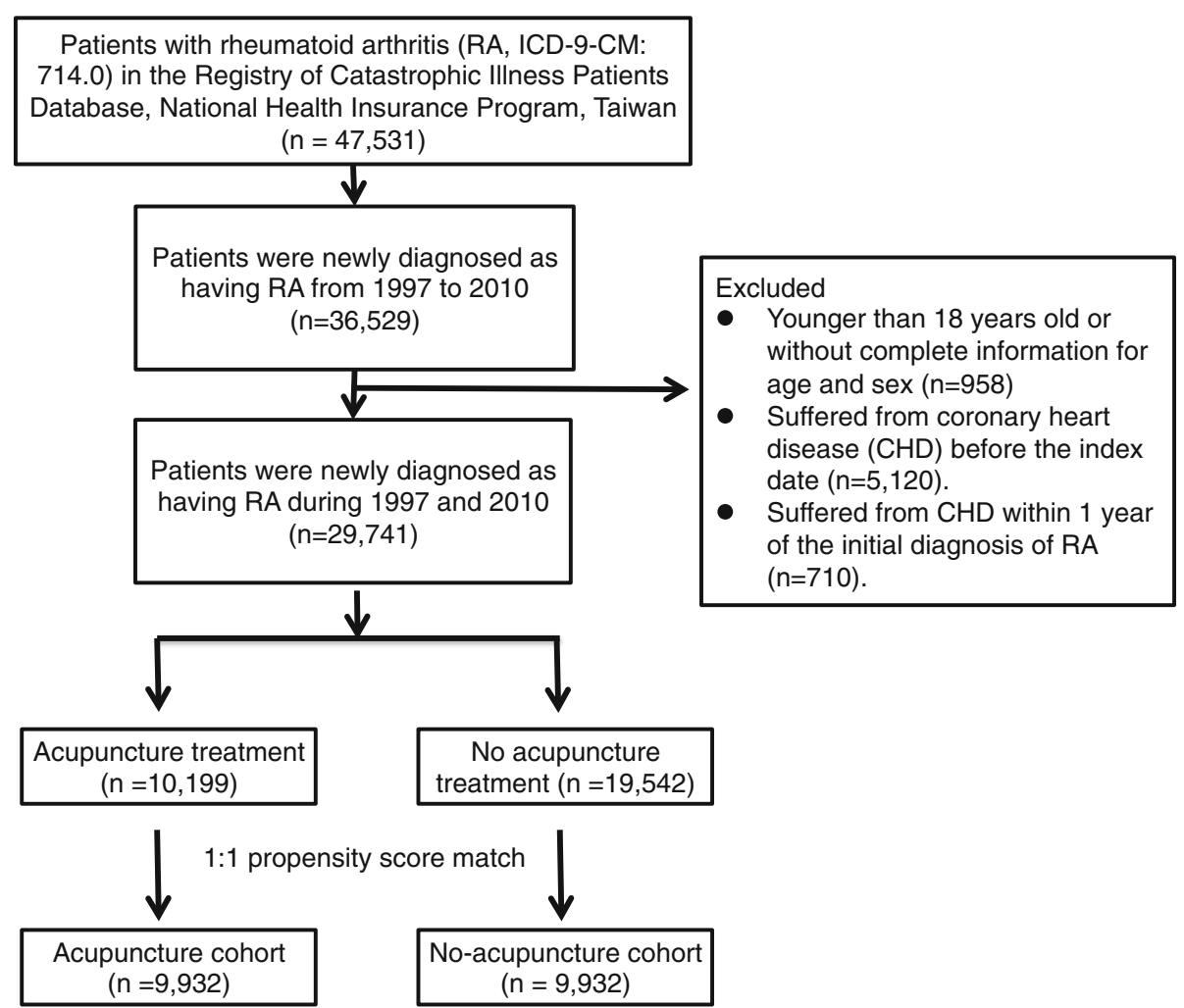

Fig. 1 Study population flowchart diagram. Using the inclusion and exclusion criteria, we identified 10,199 patients who underwent acupuncture and 19,542 patients who never underwent acupuncture. After performing 1:1 propensity score matching, there were 9932 patients in the acupuncture and no-acupuncture cohorts 
(A) Manual acupuncture

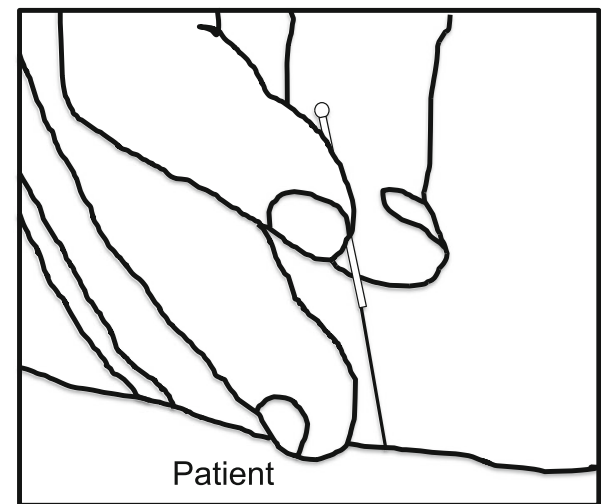

(B) Electroacupuncture

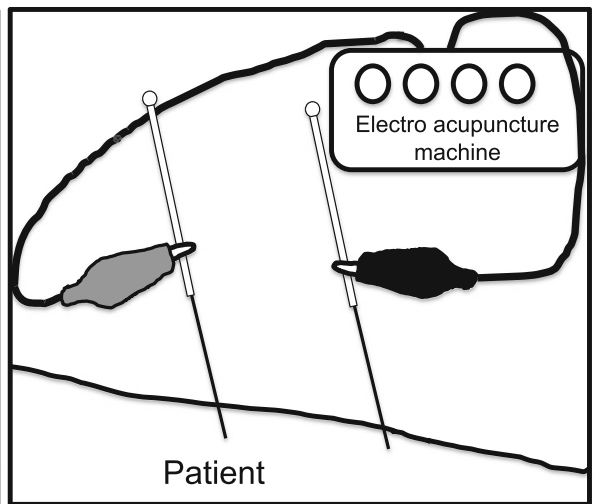

Fig. 2 Diagram of the two types of acupuncture treatment. a Manual acupuncture of traditional Chinese medicine type (b) Electroacupuncture

commonly used diagnostic codes. Because the NHIRD contains identified secondary data for research, the present study was waived from informed consent. This study was approved by the Research Ethics Committee of China Medical University and Hospital, Taichung, Taiwan (CMUH104-REC2-115).

\section{Study cohort identification and covariates}

Patients who were newly diagnosed with RA (ICD-9-CM code 714.0) between January 1, 1997 and December 31, 2010 were identified, as shown in Fig. 1. Patients younger than 18 years old or with incomplete information on the age and sex were excluded. Patients who received acupuncture between the initial diagnosis of RA and happening of CHD or December 31, 2010 were identified as the acupuncture cohort. In the acupuncture cohort, we defined the index date as the first time that the patients started to receive acupuncture. The immortal time of the acupuncture cohort was the period between the initial diagnosis of RA and the index date. Patients who suffered from CHD were identified through ICD-9-CM codes 410, 411, 413, 414.0, 414.8, and 414.9. Patients diagnosed as having CHD before the index date or within one year after their initial diagnosis of RA were excluded. Patients were followed up until December 31, 2011 or withdrawn from the NHIRD.

To reduce confounding factors, we used 1:1 propensity-score matching by sex, age (per 5 years), comorbidity, conventional drug use, the initial diagnostic year of RA, and the index year through multiple logistic regression analysis. The comorbidities analyzed in our study included DM (ICD-9-CM: 250), hypertension (ICD-9-CM: 401-405), hyperlipidemia (ICD-9-CM: 272), congestive heart failure (ICD-9-CM: 402.01, 402.11, 402.91, 404.01, 404.03, 404.11, 404.13, 404.91, 404.93, and 428.0), cerebral vascular diseases (ICD-9-CM: 430-438), depression (ICD-9-CM: 296.2-296.3, 300.4, and 311), anxiety (ICD-9-CM: 300.0, 300.2, 300.3, 308.3, and 308.91), alcoholism (ICD-9-CM: 291, 303, 305.00-305.03, 790.3, and V11.3), tobacco dependence (ICD-9-CM: 305.1), and obesity (ICD-9-CM: 278 and A183). Patients with comorbidity were identified if the ICD-9-CM codes of the selected comorbidity appeared two or more times in the outpatient or inpatient claims. The conventional drugs analyzed in our study included MTX, hydroxychloroquine, sulfasalazine, TNF- $\alpha$ inhibitors, oral steroids, NSAIDs, and statins. Finally, equal numbers of patients in the acupuncture and no-acupuncture cohorts were analyzed in this study. Patients were divided into 3 groups according to their age: $18-39$ years old, $40-59$ years old, and older than 60 years old.

\section{Types of acupuncture and disease categories in the acupuncture cohort}

We analyzed the acupuncture type that patients received by the treatment codes, which included manual acupuncture of TCM type (B41, B42, B45, B46, B80-B84, B90-B94, P27041, P31103, P32103, and P33031) and electroacupuncture (B43, B44, B86-89, and P33032). Figure 2 illustrated these two types of acupuncture treatments.

\section{Statistical analyses}

Statistical analysis was performed using SAS 9.4 (SAS Institute, Cary, NC, U.S.A.), and $p<0.05$ in two-tailed tests indicate statistical significance. The difference of baseline characteristics between the acupuncture and no-acupuncture cohorts was expressed as standardized mean differences. Standardized mean differences with less than $0.2 \mathrm{~s}$.d. indicated a negligible difference in the mean or proportions between the two cohorts. For each variable, we used Cox proportional hazard regression to analyze the hazard ratios (HRs) and 95\% confidence interval (95\% CI). The Kaplan-Meier method was used 
to determine the cumulative incidence of CHD in both cohorts, and the log-rank test was used to compare incidence curves between the acupuncture and no-acupuncture cohorts.

\section{Results}

The identified patient numbers in both cohorts were equal after performing the 1:1 propensity score matched method (Fig. 1). There were no significant differences in the sex, age, baseline comorbidity, and conventional drug use between the no-acupuncture and acupuncture cohorts (Table 1). The included patients were female predominant with a mean age at approximately 50 years old in both cohorts. Almost all patients in both cohorts have used NSAIDs, and more than $90 \%$ of patients have also used oral steroids.

Table 1 Characteristics of rheumatoid arthritis patients according to whether they received acupuncture treatment

\begin{tabular}{|c|c|c|c|c|c|}
\hline \multirow[t]{3}{*}{ Variable } & \multicolumn{4}{|c|}{$\begin{array}{l}\text { Rheumatoid arthritis } \\
\text { Underwent acupuncture }\end{array}$} & \multirow{3}{*}{$\begin{array}{l}\text { Standardizec } \\
\text { mean } \\
\text { difference }\end{array}$} \\
\hline & \multicolumn{2}{|c|}{ No $(n=9932)$} & \multicolumn{2}{|c|}{ Yes $(n=9932)$} & \\
\hline & $\bar{n}$ & $\%$ & $\bar{n}$ & $\%$ & \\
\hline \multicolumn{6}{|l|}{$\overline{\text { Sex }}$} \\
\hline Female & 8212 & 82.68 & 8225 & 82.81 & 0.003 \\
\hline Male & 1720 & 17.32 & 1707 & 17.19 & 0.003 \\
\hline \multicolumn{6}{|l|}{ Age group } \\
\hline $18-39$ & 2267 & 22.83 & 2013 & 20.27 & 0.003 \\
\hline $40-59$ & 6099 & 61.41 & 6659 & 67.05 & 0.003 \\
\hline$\geq 60$ & 1566 & 15.77 & 1260 & 12.69 & 0.003 \\
\hline Mean \pm SD (years) & \multicolumn{2}{|c|}{$50.39(13.7)$} & \multicolumn{2}{|c|}{$50.36(12.53)$} & 0.002 \\
\hline \multicolumn{6}{|l|}{ Baseline Comorbidity } \\
\hline Diabetes mellitus & 1211 & 12.19 & 1193 & 12.01 & 0.006 \\
\hline Hypertension & 2358 & 23.74 & 2339 & 23.55 & 0.005 \\
\hline Hyperlipidemia & 1488 & 14.98 & 1475 & 14.85 & 0.004 \\
\hline Congestive heart failure & 94 & 0.95 & 107 & 1.08 & 0.013 \\
\hline Stroke & 741 & 7.46 & 731 & 7.36 & 0.004 \\
\hline Depression & 631 & 6.35 & 618 & 6.22 & 0.005 \\
\hline Anxiety & 1525 & 15.35 & 1499 & 15.09 & 0.007 \\
\hline Alcoholism & 20 & 0.20 & 24 & 0.24 & 0.009 \\
\hline Tobacco dependence & 27 & 0.27 & 30 & 0.30 & 0.006 \\
\hline Obesity & 80 & 0.81 & 77 & 0.78 & 0.003 \\
\hline \multicolumn{6}{|l|}{ Drug use } \\
\hline Methotrexate & 7131 & 71.80 & 7130 & 71.79 & 0 \\
\hline Hydroxychloroquine & 8372 & 84.29 & 8423 & 84.81 & 0.014 \\
\hline Sulfasalazine & 7289 & 73.39 & 7278 & 73.28 & 0.003 \\
\hline TNF-a inhibitors & 1137 & 11.45 & 1151 & 11.59 & 0.004 \\
\hline NSAIDs & 9908 & 99.76 & 9909 & 99.77 & 0.002 \\
\hline Oral steroids & 9449 & 95.14 & 9438 & 95.03 & 0.005 \\
\hline Statins & 1449 & 14.59 & 1438 & 14.48 & 0.003 \\
\hline \multicolumn{6}{|l|}{ Types of acupuncture } \\
\hline Manual acupuncture of TCM type & & & 8614 & 86.73 & \\
\hline Electroacupuncture & & & 340 & 3.42 & \\
\hline Combination of manual acupuncture and electroacupuncture & & & 978 & 9.85 & \\
\hline Immortal time, days (mean, median) & \multicolumn{2}{|c|}{$1064.07(989.63)$} & \multicolumn{2}{|c|}{$1044.96(1056.50)$} & \\
\hline Acupuncture visits, mean & & & \multicolumn{2}{|l|}{9.81} & \\
\hline Follow-up time, years (mean, median) & \multicolumn{2}{|c|}{$4.02(3.03)$} & \multicolumn{2}{|c|}{$4.91(3.44)$} & \\
\hline
\end{tabular}


Approximately $15 \%$ of patients took statins in both the no-acupuncture and acupuncture cohorts. Manual acupuncture of the TCM type was the most commonly used acupuncture treatment.

During the follow-up period, 909 patients in the acupuncture cohort and 1233 patients in the no-acupuncture cohort developed CHD (Table 2). Compared with the no-acupuncture cohort, the incidence of CHD was significantly lower in the acupuncture cohort (adjusted $\mathrm{HR}=0.60,95 \% \mathrm{CI}=0.55-0.65$ ). RA patients with old age, DM, or hypertension were prone to developing CHD.
The beneficial effect of acupuncture on the incidence of CHD was observed in both women and men (Table 3, adjusted $\mathrm{HR}=0.62$ in women, $95 \% \mathrm{CI}=0.56-0.68$; adjusted $\mathrm{HR}=0.58$ in men, $95 \% \mathrm{CI}=0.48-0.70$ ). Acupuncture significantly decreased the incidence of CHD in all age groups. Regardless of whether patients had DM, hypertension, hyperlipidemia, or stroke, acupuncture lowered the risk of CHD. Moreover, the beneficial effect of acupuncture in preventing CHD was independent of conventional drug use, including statins, MTX, hydroxychloroquine, sulfasalazine, oral steroids, and TNF- $\alpha$ inhibitors. Overall, the cumulative

Table 2 Cox model with hazard ratios and 95\% confidence intervals for coronary heart disease in patients with rheumatoid arthritis who did or did not receive acupuncture treatment

\begin{tabular}{|c|c|c|c|c|c|c|c|}
\hline \multirow[t]{2}{*}{ Variable } & \multirow{2}{*}{$\begin{array}{l}\text { No. of event } \\
(n=2142)\end{array}$} & \multicolumn{3}{|c|}{ Crude $^{a}$} & \multicolumn{3}{|c|}{ Adjusted $^{b}$} \\
\hline & & $\mathrm{HR}$ & $(95 \% \mathrm{Cl})$ & $p$-value & $\mathrm{HR}$ & $(95 \% \mathrm{Cl})$ & $p$-value \\
\hline \multicolumn{8}{|l|}{ Acupuncture } \\
\hline No & 1233 & 1.00 & reference & & 1.00 & reference & \\
\hline Yes & 909 & 0.63 & $(0.58-0.69)$ & $<.0001$ & 0.60 & $(0.55-0.65)$ & $<.0001$ \\
\hline \multicolumn{8}{|l|}{ Sex } \\
\hline Female & 1692 & 1.00 & reference & & 1.00 & reference & \\
\hline Male & 440 & 1.32 & $(1.19-1.47)$ & $<.0001$ & 1.21 & $(1.09-1.34)$ & 0.0005 \\
\hline \multicolumn{8}{|l|}{ Age group } \\
\hline $18-39$ & 126 & 1.00 & reference & & 1.00 & reference & \\
\hline $40-59$ & 1458 & 4.22 & $(3.52-5.07)$ & $<.0001$ & 3.85 & $(3.20-4.63)$ & $<.0001$ \\
\hline$\geq 60$ & 548 & 8.76 & $(7.22-10.64)$ & $<.0001$ & 5.35 & $(4.37-6.56)$ & $<.0001$ \\
\hline \multicolumn{8}{|c|}{ Baseline Comorbidity (ref = non-site comorbidity) } \\
\hline Diabetes mellitus & 443 & 2.25 & $(2.03-2.50)$ & $<.0001$ & 1.46 & $(1.30-1.64)$ & $<.0001$ \\
\hline Hypertension & 877 & 2.65 & $(2.43-2.89)$ & $<.0001$ & 1.77 & $(1.61-1.95)$ & $<.0001$ \\
\hline Hyperlipidemia & 391 & 1.64 & $(1.47-1.83)$ & $<.0001$ & 1.08 & $(0.95-1.22)$ & 0.2342 \\
\hline Congestive heart failure & 33 & 1.94 & $(1.37-2.73)$ & 0.0002 & 1.00 & $(0.71-1.42)$ & 0.9783 \\
\hline Cerebral vascular diseases & 267 & 2.09 & $(1.84-2.38)$ & $<.0001$ & 1.22 & $(1.07-1.40)$ & 0.0037 \\
\hline Depression & 127 & 1.18 & $(0.99-1.42)$ & 0.0664 & 0.97 & $(0.80-1.17)$ & 0.7509 \\
\hline Anxiety & 312 & 1.20 & $(1.07-1.36)$ & 0.0028 & 0.94 & $(0.83-1.07)$ & 0.3402 \\
\hline Alcoholism & 2 & 0.54 & $(0.14-2.17)$ & 0.3886 & 0.51 & $(0.13-2.05)$ & 0.3427 \\
\hline Tobacco dependence & 1 & 0.25 & $(0.04-1.74)$ & 0.1606 & 0.22 & $(0.03-1.57)$ & 0.1316 \\
\hline Obesity & 18 & 1.38 & $(0.87-2.20)$ & 0.171 & 1.15 & $(0.72-1.83)$ & 0.5623 \\
\hline \multicolumn{8}{|l|}{ Drug use } \\
\hline Methotrexate & 1210 & 0.47 & $(0.43-0.51)$ & $<.0001$ & 0.64 & $(0.58-0.70)$ & $<.0001$ \\
\hline Hydroxychloroquine & 1680 & 0.60 & $(0.55-0.67)$ & $<.0001$ & 0.80 & $(0.720-.89)$ & $<.0001$ \\
\hline Sulfasalazine & 1437 & 0.66 & $(0.61-0.73)$ & $<.0001$ & 0.91 & $(0.82-1.00)$ & 0.0559 \\
\hline TNF-a inhibitors & 105 & 0.35 & $(0.29-0.43)$ & $<.0001$ & 0.48 & $(0.39-0.59)$ & $<.0001$ \\
\hline NSAIDs & 2130 & 1.36 & $(0.34-5.44)$ & 0.6638 & 1.73 & $(0.43-6.93)$ & 0.4412 \\
\hline Oral steroids & 2010 & 0.66 & $(0.55-0.79)$ & $<.0001$ & 0.82 & $(0.68-0.98)$ & 0.033 \\
\hline Statins & 350 & 1.07 & $(0.96-1.20)$ & 0.2408 & 0.69 & $(0.61-0.78)$ & $<.0001$ \\
\hline
\end{tabular}

Crude $\mathrm{HR}^{\mathrm{a}}$ represents the relative hazard ratio. Adjusted $\mathrm{HR}^{\mathrm{b}}$ represents the adjusted hazard ratio mutually adjusted for acupuncture use, age, sex, diabetes mellitus, hypertension, hyperlipidemia, congestive heart failure, cerebral vascular diseases, depression, anxiety, alcoholism, tobacco dependence, obesity, Methotrexate, Hydroxychloroquine, Sulfasalazine, TNF-a inhibitors, oral steroids, NSAIDs and statins in Cox proportional hazard regression 
Table 3 Incidence rates, hazard ratio and confidence intervals for coronary heart disease in rheumatoid arthritis patients who did or did not receive acupuncture treatment, stratified by sex, age, comorbidity and drug use

\begin{tabular}{|c|c|c|c|c|c|c|c|c|}
\hline \multirow[t]{3}{*}{ Variables } & \multicolumn{6}{|c|}{ Rheumatoid arthritis } & \multicolumn{2}{|c|}{ Compared with no-acupuncture cohort } \\
\hline & \multicolumn{3}{|c|}{$\begin{array}{l}\text { No acupuncture } \\
(n=9932)\end{array}$} & \multicolumn{3}{|c|}{$\begin{array}{l}\text { Acupuncture } \\
(n=9932)\end{array}$} & \multirow[t]{2}{*}{$\begin{array}{l}\text { Crude HR } \\
(95 \% \mathrm{Cl})\end{array}$} & \multirow[t]{2}{*}{$\begin{array}{l}\text { Adjusted HR } \\
(95 \% \mathrm{Cl})\end{array}$} \\
\hline & Event & Person-years & $\mathrm{IR}$ & Event & Person-years & $\mathrm{IR}$ & & \\
\hline Total & 1223 & $39,930.25$ & 30.63 & 909 & $48,742.08$ & 18.65 & $0.63(0.58-0.69)^{* * *}$ & $0.60(0.55-0.65)^{* * *}$ \\
\hline \multicolumn{9}{|l|}{ Sex } \\
\hline Female & 970 & $33,542.81$ & 28.92 & 722 & $40,610.75$ & 17.78 & $0.64(0.58-0.7)^{* * *}$ & $0.62(0.56-0.68)^{* * *}$ \\
\hline Male & 253 & 6387.44 & 39.61 & 187 & 8131.32 & 23.00 & $0.60(0.50-0.73)^{* * *}$ & $0.58(0.48-0.70)^{* * *}$ \\
\hline \multicolumn{9}{|l|}{ Age group } \\
\hline $18-39$ & 81 & $10,544.53$ & 7.68 & 45 & $10,665.24$ & 4.22 & $0.56(0.39-0.81)^{* *}$ & $0.53(0.37-0.77)^{* * *}$ \\
\hline $40-59$ & 816 & $24,725.99$ & 33.00 & 642 & $32,628.29$ & 19.68 & $0.61(0.55-0.68)^{* * * *}$ & $0.59(0.53-0.66)^{* * *}$ \\
\hline$\geq 60$ & 326 & 4659.72 & 69.96 & 222 & 5448.55 & 40.74 & $0.61(0.51-0.72)^{* * *}$ & $0.62(0.52-0.73)^{* * *}$ \\
\hline
\end{tabular}

Baseline Comorbidity

Diabetes mellitus

$\begin{array}{lllllllll}\text { No } & 969 & 36,055.56 & 26.88 & 720 & 43,658.67 & 16.49 & 0.64(0.58-0.70)^{* * *} & 0.60(0.55-0.66)^{* * * *} \\ \text { Yes } & 254 & 3874.69 & 65.55 & 189 & 5083.41 & 37.18 & 0.58(0.48-0.71)^{* * *} & 0.59(0.49-0.72)^{* * *}\end{array}$

Hypertension

$\begin{array}{lllllllll}\text { No } & 717 & 32,065.40 & 22.36 & 538 & 38,485.07 & 13.98 & 0.64(0.57-0.72)^{* * *} & 0.62(0.56-0.70)^{* * *} \\ \text { Yes } & 506 & 7864.85 & 64.34 & 371 & 10,257.00 & 36.17 & 0.59(0.51-0.67)^{* * *} & 0.58(0.51-0.67)^{* * *}\end{array}$

Hyperlipidemia

\begin{tabular}{|c|c|c|c|c|c|c|c|c|}
\hline No & 1000 & $35,475.99$ & 28.19 & 741 & $43,080.05$ & 17.20 & $0.63(0.58-0.70)^{* * *}$ & $0.61(0.56-0.67)^{* * *}$ \\
\hline Yes & 223 & 4454.26 & 50.06 & 168 & 5662.03 & 29.67 & $0.59(0.49-0.73)^{* * *}$ & $0.57(0.47-0.70)^{* * *}$ \\
\hline \multicolumn{9}{|c|}{ Congestive heart failure } \\
\hline No & 1202 & $39,641.02$ & 30.32 & 897 & $48,354.94$ & 18.55 & $0.63(0.58-0.69)^{* * *}$ & $0.61(0.56-0.66)^{* * *}$ \\
\hline Yes & 21 & 289.23 & 72.61 & 12 & 387.14 & 31.00 & $0.44(0.22-0.90)^{*}$ & $0.50(0.24-1.07)$ \\
\hline
\end{tabular}

Cerebral vascular diseases

$\begin{array}{lllllllll}\text { No } & 1069 & 37,549.80 & 28.47 & 796 & 45,604.09 & 17.45 & 0.64(0.58-0.70)^{* * *} & 0.60(0.55-0.66)^{* * *} \\ \text { Yes } & 154 & 2380.45 & 64.69 & 113 & 3137.99 & 36.01 & 0.58(0.45-0.74)^{* * *} & 0.59(0.46-0.76)^{* * *}\end{array}$

Depression

\begin{tabular}{|c|c|c|c|c|c|c|c|c|}
\hline No & 1144 & $38,013.07$ & 30.09 & 861 & $46,405.65$ & 18.55 & $0.64(0.59-0.70)^{* * *}$ & $0.62(0.56-0.67)^{* * *}$ \\
\hline Yes & 79 & 1917.19 & 41.21 & 48 & 2336.42 & 20.54 & $0.51(0.36-0.73)^{* * *}$ & $0.51(0.35-0.73)^{* * *}$ \\
\hline \multicolumn{9}{|c|}{ Anxiety } \\
\hline No & 1042 & $35,170.64$ & 29.63 & 778 & $43,056.56$ & 18.07 & $0.63(0.58-0.69)^{* * *}$ & $0.60(0.55-0.66)^{* * *}$ \\
\hline Yes & 181 & 4759.61 & 38.03 & 131 & 5685.51 & 23.04 & $0.62(0.50-0.78)^{* * *}$ & $0.62(0.49-0.78)^{* * *}$ \\
\hline \multicolumn{9}{|c|}{ Alcoholism } \\
\hline No & 1222 & $39,865.91$ & 1222 & 908 & $48,660.02$ & 908 & $0.63(0.58-0.69)^{* * *}$ & $0.61(0.56-0.66)^{* * *}$ \\
\hline Yes & 1 & 64.34 & 1 & 1 & 82.05 & 1 & $0.58(0.03-9.89)$ & - \\
\hline \multicolumn{9}{|c|}{ Tobacco dependence } \\
\hline No & 1222 & $39,862.01$ & 1222 & 909 & $48,661.38$ & 909 & $0.63(0.58-0.69)^{* * *}$ & $0.61(0.56-0.66)^{* * *}$ \\
\hline Yes & 1 & 68.24 & 1 & 0 & 80.70 & 0 & - & - \\
\hline \multicolumn{9}{|c|}{ Obesity } \\
\hline No & 1214 & $39,697.39$ & 30.58 & 900 & $48,463.26$ & 18.57 & $0.63(0.58-0.69)^{* * *}$ & $0.61(0.55-0.66)^{* * *}$ \\
\hline Yes & 9 & 232.86 & 38.65 & 9 & 278.81 & 32.28 & $0.95(0.37-2.42)$ & $0.74(0.26-2.09)$ \\
\hline
\end{tabular}


Table 3 Incidence rates, hazard ratio and confidence intervals for coronary heart disease in rheumatoid arthritis patients who did or did not receive acupuncture treatment, stratified by sex, age, comorbidity and drug use (Continued)

\begin{tabular}{|c|c|c|c|c|c|c|c|c|}
\hline \multirow[t]{3}{*}{ Variables } & \multicolumn{6}{|c|}{ Rheumatoid arthritis } & \multicolumn{2}{|c|}{ Compared with no-acupuncture cohort } \\
\hline & \multicolumn{3}{|c|}{$\begin{array}{l}\text { No acupuncture } \\
(n=9932)\end{array}$} & \multicolumn{3}{|c|}{$\begin{array}{l}\text { Acupuncture } \\
(n=9932)\end{array}$} & \multirow[t]{2}{*}{$\begin{array}{l}\text { Crude HR } \\
(95 \% \mathrm{Cl})\end{array}$} & \multirow[t]{2}{*}{$\begin{array}{l}\text { Adjusted HR } \\
(95 \% \mathrm{Cl})\end{array}$} \\
\hline & Event & Person-years & IR & Event & Person-years & $\mathrm{IR}$ & & \\
\hline \multicolumn{9}{|l|}{ Drug use } \\
\hline \multicolumn{9}{|c|}{ Methotrexate } \\
\hline No & 530 & $10,045.02$ & 52.76 & 392 & $13,042.58$ & 30.06 & $0.60(0.53-0.68)^{* * *}$ & $0.61(0.54-0.7)^{* * *}$ \\
\hline Yes & 693 & $29,885.23$ & 23.19 & 517 & $35,699.50$ & 14.48 & $0.64(0.57-0.72)^{* * *}$ & $0.60(0.53-0.67)^{* * *}$ \\
\hline \multicolumn{9}{|c|}{ Hydroxychloroquine } \\
\hline No & 257 & 5500.43 & 46.72 & 195 & 6736.19 & 28.95 & $0.65(0.54-0.78)^{* * *}$ & $0.62(0.52-0.75)^{* * *}$ \\
\hline Yes & 966 & $34,429.82$ & 28.06 & 714 & $42,005.89$ & 17 & $0.63(0.57-0.69)^{* * *}$ & $0.60(0.54-0.66)^{* * *}$ \\
\hline \multicolumn{9}{|c|}{ Sulfasalazine } \\
\hline No & 410 & 9296.59 & 44.10 & 285 & $11,957.00$ & 23.84 & $0.57(0.49-0.66)^{* * *}$ & $0.54(0.47-0.63)^{* * *}$ \\
\hline Yes & 813 & $30,633.66$ & 26.54 & 624 & $36,785.07$ & 16.96 & $0.66(0.59-0.73)^{* * *}$ & $0.64(0.57-0.71)^{* * *}$ \\
\hline \multicolumn{9}{|c|}{ TNF-a inhibitors } \\
\hline No & 1157 & $34,731.45$ & 33.31 & 870 & $42,448.62$ & 20.50 & $0.64(0.59-0.7)^{* * *}$ & $0.62(0.56-0.67)^{* * *}$ \\
\hline Yes & 66 & 5198.80 & 12.70 & 39 & 6293.45 & 6.20 & $0.48(0.32-0.72)^{* * *}$ & $0.42(0.28-0.62)^{* * *}$ \\
\hline \multicolumn{9}{|l|}{ NSAIDs } \\
\hline No & 0 & 42.45 & 0 & 2 & 62.67 & 31.91 & - & - \\
\hline Yes & 1223 & $39,887.80$ & 30.66 & 907 & $48,679.41$ & 18.63 & $0.63(0.58-0.69)^{* * *}$ & $0.61(0.56-0.66)^{* * *}$ \\
\hline \multicolumn{9}{|c|}{ Oral steroids } \\
\hline No & 68 & 1442.40 & 47.14 & 54 & 1799.74 & 30 & $0.69(0.48-1.00)^{*}$ & $0.74(0.51-1.07)$ \\
\hline Yes & 1155 & $38,487.85$ & 30.01 & 855 & $46,942.34$ & 18.21 & $0.63(0.57-0.69)^{* * *}$ & $0.60(0.55-0.65)^{* * *}$ \\
\hline \multicolumn{9}{|l|}{ Statins } \\
\hline No & 1020 & $33,516.65$ & 30.43 & 762 & $41,308.09$ & 18.45 & $0.63(0.58-0.69)^{* * *}$ & $0.61(0.55-0.67)^{* * *}$ \\
\hline Yes & 203 & 6413.60 & 31.65 & 147 & 7433.99 & 19.77 & $0.63(0.51-0.78)^{* * *}$ & $0.59(0.48-0.73)^{* * *}$ \\
\hline
\end{tabular}

Abbreviations: IR, incidence rates per 1000 person-years; $\mathrm{HR}$, hazard ratio; and $\mathrm{Cl}$, confidence interval

Adjusted HR: adjusted for acupuncture use, age, sex, diabetes mellitus, hypertension, hyperlipidemia, congestive heart failure, cerebral vascular diseases,

depression, anxiety, alcoholism, tobacco dependence, obesity, Methotrexate, Hydroxychloroquine, Sulfasalazine, TNF-a inhibitors, oral steroids, NSAIDs and statins in Cox proportional hazard regression

*: $p<0.05 ;{ }^{* *}: p<0.01$; and ${ }^{* * *}: p<0.001$

incidence of CHD from the index date was significantly lower in the acupuncture cohort (Fig. 3).

Subgroup analysis was performed to evaluate whether different types of acupuncture had any correlation and difference on preventing CHD in patients with RA (Table 4). Patients receiving only manual acupuncture, only electroacupuncture, or combination of manual acupuncture and electroacupuncture all had a lower incidence of CHD than patients never receiving acupuncture.

Among the different types of CHD, patients with acupuncture had a significant lower incidence of chronic ischemic heart disease (Table 5). However, there was no significant difference in the incidence of angina pectoris between the two groups. The effect on acute myocardial infarction was not analyzed (data not shown; only 11 patients had acute myocardial infarction in two groups).
Table 6 showed the top five disease categories, which represented the reasons for clinical visits, in the acupuncture cohort. Most patients with RA received acupuncture because of musculoskeletal disease.

\section{Discussion}

To the best of our knowledge, this nationwide population-based study is the first report to reveal that acupuncture decreased the risk of CHD in patients with RA. Our study found that the beneficial effects of acupuncture for developing CHD in patients with RA were independent of sex, age, DM, and hypertension. Moreover, acupuncture decreased the risk of CHD in patients with RA who took statins, which was reported to reduce the risk of cardiovascular diseases [20]. Among different types of CHD, our results demonstrated that patients with acupuncture had a significant lower incidence of 


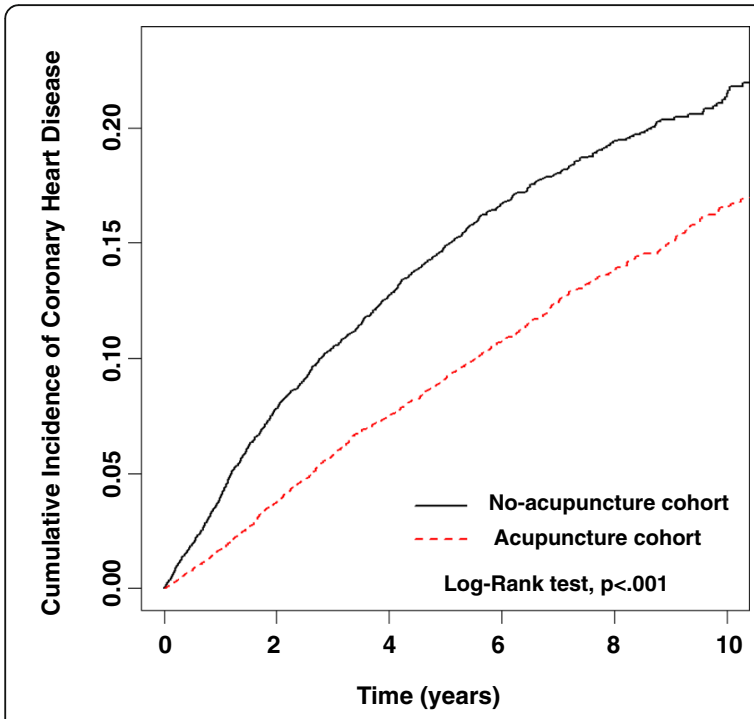

Fig. 3 Cumulative incidence of coronary heart disease (CHD) between the acupuncture and no-acupuncture cohorts. The cumulative incidence of coronary heart disease in the acupuncture cohort (dashed line) is significantly lower than the no-acupuncture cohort (solid line) (log-rank test, $p<.001$ ). The zero point indicated the index date

chronic ischemic heart disease than patients without receiving acupuncture treatment.

The risk of CHD in patients with RA is highly associated with the conventional risk factors of CHD, such as hypertension, DM, hyperlipidemia, and physical inactivity [6]. Our study also demonstrated that RA patients with comorbidities, including DM, hypertension, or hyperlipidemia, had a higher risk of CHD. Even in RA patients with DM, hypertension, or hyperlipidemia, our study indicated that acupuncture decreased the incidence of CHD. Previous clinical trials indicated that acupuncture improved swelling and range of motion of knee joints, physical activity, and quality of life in patient with RA [14, 21, 22]. Improved physical activity might be one of the possible explanations that acupuncture can prevent CHD in patients with RA. However, we also found that for those RA patients with the comorbidity of congestive heart failure, acupuncture did not reduce the risk of CHD.

The previous study stated that endothelial dysfunction contributed to atherosclerosis and induced cardiovascular disorders [23]. The current conventional drugs for RA treatment, including glucocorticoids [24] and etanercept (a TNF- $\alpha$ inhibitor) [9], have been demonstrated to improve endothelial dysfunction through the anti-inflammatory effect in RA rat models. However, the protective effect of glucocorticoids remains controversial because glucocorticoids also enhance the risk of cardiovascular disorders based on their negative effects on blood pressure, lipid, insulin resistance and obesity [25]. The protective effect of MTX at a low dose against CHD in patients with RA was also demonstrated in a previous meta-analysis study although it did not attenuate hyperlipidemia or insulin resistance [8]. Our study also indicated that patients with RA who took MTX or TNF- $\alpha$ inhibitors had a lower risk of CHD. In addition, regardless of whether patients used TNF- $\alpha$ inhibitors or MTX, acupuncture significantly decreased the risk of CHD in those patients.

Chronic inflammation plays an important role in the pathogenesis of RA and its comorbidities, including DM, hypertension, and CHD [7]. As a result, amelioration of inflammatory activity should be considered in the CHD preventive strategy for patients with RA. The anti-inflammatory mechanisms of acupuncture have been evaluated in murine models of inflammatory disorders [11, 12]. Acupuncture corrected the imbalance between Th17 and Treg cells to attenuate the severity of RA in a rat RA model [26]. IL-17, cytokine secreted by Th17 cells, has been demonstrated to be important in the pathogenesis of RA, and it has been demonstrated to be associated with CHD [27]. Further mechanistic studies are needed to evaluate whether acupuncture can suppress IL-17 in RA to prevent CHD. Previous clinical trials have demonstrated that acupuncture suppressed inflammation in patients with RA [13, 14]. Based on these studies, acupuncture not only had a traditionally

Table 4 Cox model for coronary heart disease in patients with rheumatoid arthritis receiving different types of acupuncture treatment vs. not receiving acupuncture treatment

\begin{tabular}{lllll}
\hline & $n$ & $\begin{array}{l}\text { CHD } \\
(n=2132)\end{array}$ & Crude HR (95\%Cl) & Adjusted HR ${ }^{\dagger}(95 \% \mathrm{Cl})$ \\
\hline No-acupuncture & 9932 & 1223 & 1 (reference) & 1 (reference) \\
$\begin{array}{l}\text { Acupuncture } \\
\text { Manual acupuncture of TCM type }\end{array}$ & 8614 & 819 & $0.67(0.62-0.74)^{* * *}$ & $0.64(0.58-0.69)^{* * *}$ \\
Electroacupuncture & 340 & 19 & $0.48(0.31-0.76)^{* *}$ & $0.46(0.29-0.73)^{* *}$ \\
Combination of manual acupuncture and electroacupuncture & 978 & 71 & $0.39(0.30-0.49)^{* * *}$ & $0.36(0.28-0.46)^{* * *}$ \\
\hline
\end{tabular}

Abbreviations: $\mathrm{HR}$, hazard ratio; $\mathrm{CHD}$, coronary heart disease; $\mathrm{Cl}$, confidence interval; and TCM, traditional Chinese medicine

Crude $\mathrm{HR}^{*}$ represents relative hazard ratio. Adjusted $\mathrm{HR}^{\dagger}$ represents adjusted hazard ratio mutually adjusted for age, sex, diabetes mellitus, hypertension,

hyperlipidemia, congestive heart failure, cerebral vascular diseases, depression, anxiety, alcoholism, tobacco dependence, obesity, Methotrexate,

Hydroxychloroquine, Sulfasalazine, TNF-a inhibitors, oral steroids, NSAIDs and statins in Cox proportional hazard regression

**: $p<0.01$; and ${ }^{* * *}: p<0.001$ 
Table 5 Cox model for angina pectoris and chronic ischemic heart disease in patients with rheumatoid arthritis receiving acupuncture treatment vs. not receiving acupuncture treatment

\begin{tabular}{|c|c|c|c|c|c|c|c|}
\hline & \multirow[t]{2}{*}{$\mathrm{N}$} & \multicolumn{3}{|c|}{ Angina pectoris } & \multicolumn{3}{|c|}{ Chronic ischemic heart disease } \\
\hline & & $\bar{n}$ & Crude $H R^{*}$ & $\overline{\text { Adjusted } \mathrm{HR}^{\dagger}}$ & $\bar{n}$ & Crude HR* $(95 \% \mathrm{Cl})$ & Adjusted $\mathrm{HR}^{\dagger}(95 \% \mathrm{Cl})$ \\
\hline \multicolumn{8}{|c|}{ Acupuncture } \\
\hline No & 8733 & 24 & 1 (reference) & 1 (reference) & 516 & 1 (reference) & 1 (reference) \\
\hline Yes & 9044 & 21 & $0.79(0.44-1.43)$ & $0.72(0.4-1.3)$ & 358 & $0.59(0.52-0.68)^{* * *}$ & $0.56(0.49-0.64)^{* * *}$ \\
\hline
\end{tabular}

Crude $\mathrm{HR}^{*}$ represents relative hazard ratio. Adjusted $\mathrm{HR}^{\dagger}$ represents adjusted hazard ratio mutually adjusted for age, sex, diabetes mellitus, hypertension, hyperlipidemia, congestive heart failure, cerebral vascular diseases, depression, anxiety, alcoholism, tobacco dependence, obesity, Methotrexate, Hydroxychloroquine, Sulfasalazine, TNF-a inhibitors, oral steroids, NSAIDs and statins in Cox proportional hazard regression $* * *: p<0.001$

recognized analgesic effect, it had an anti-inflammatory effect. Anti-inflammation might be one of the mechanisms by which acupuncture decreased the incidence of CHD in RA patients. In addition, previous studies demonstrated that the combination of acupuncture with current conventional treatment had additive effects on hypertensive patients [28] and diabetic patients [29], which could contribute to how acupuncture decreased the risk of CHD in RA patients with comorbidity or conventional treatment. The mechanistic study is needed in the future. We have established a collagen-induced arthritis murine model and designed a clinical trial to investigate whether acupuncture is effective on the immunological modulation in RA.

The strength of this study was the comprehensive large-scale database, Taiwan's NHIRD, which provides a large sample size and reduces selection bias while including long-term follow-up data [17]. Although there were a few clinical trials investigating the efficacy of acupuncture for the treatment of patients with RA [13, 14, $21,22,30-35]$, none of them addressed the question whether acupuncture treatment reduces the risk of cardiovascular disease among patients with RA.

Some limitations merit attention in our study. First, the detailed information about severity of RA, including pain scores, the severity of inflammation, or the destruction of cartilage and bone were not provided in the database. Because patients with different severities may receive different conventional treatments, we performed a 1:1 propensity score matching to minimize this confounding factor. We found that there were no difference in percentages of patients who used analgesic drugs, including NSAIDs and oral steroids, in the acupuncture and no-acupuncture cohorts. The second limitation is that lifestyle data, such as smoking, alcohol drinking, and body mass index (BMI), were unavailable in the datasets. We were able to acquire information of the illnesses that resulted from these personal habits and lifestyles, including alcoholism, tobacco dependence, and obesity. However, an underestimation of smoking exposure and alcohol drinking in both cohorts still may exist. The third limitation was that the datasets did not provide detailed information of selected acupoints and treatment protocols. Usually, TCM doctors selected acupoints for each patient according to the TCM diagnosis, which may vary during the disease progression. In addition, the duration or visit times of acupuncture in the current study might be underestimated because the NHI program only covers maximal 15 times of acupuncture per month and maximal 2-3 times per week, some patients may use self-pay for additional acupuncture treatment, which could not be identified from the NHIRD database. Future clinical trials should consider comparing the effects of a set of fixed acupoints versus flexible acupoints and different duration/times of acupuncture treatment. Although there are some limitations in the current retrospective cohort study, it's a "real-world evidence" that can reflect actual use in practice [36] from a comprehensive large-scale database. Evidences gathered from future clinical trials should be able to provide more information regarding the selection of acupoints.

Table 6 The top five disease categories (as the reasons for clinical visits) in acupuncture user cohort

\begin{tabular}{lll}
\hline Disease (ICD-9-CM) & $\begin{array}{l}\text { Acupuncture users } \\
(n=9932)\end{array}$ & $\%$ \\
\cline { 2 - 3 } & $\mathrm{n}$ & 69.32 \\
\hline Musculoskeletal system and connective tissue (710-739) & 6885 & 54.14 \\
Injury (800-999) & 5377 & 7.05 \\
Symptoms, signs and ill-defined conditions (780-799) & 700 & 4.66 \\
Nervous system (320-389) & 463 & 3.04 \\
Digestive system (520-579) & 302 & \\
\hline
\end{tabular}

Maximal of three ICD-9-CM codes could be recorded in one visit 


\section{Conclusions}

Our study revealed that acupuncture treatment might have beneficial effect on reducing the risk of CHD in patients with RA in Taiwan. This noteworthy finding can provide useful information for further clinical and mechanistic studies.

\section{Abbreviations \\ 95\% Cl: 95\% confidence interval; CHD: Coronary heart disease; DM: Diabetes mellitus; DMARDs: Disease-modifying anti-rheumatic drugs; HRs: Hazard ratios; ICD-9-CM: International Classification of Diseases, Ninth Revision, Clinical Modification; MTX: Methotrexate; NHI: National Health Insurance; NHIRD: National Health Insurance Research Database; NSAIDs: Non-steroid anti-inflammatory drugs; RA: Rheumatoid arthritis; TCM: Traditional Chinese medicine}

\section{Acknowledgements}

This study was based in part on data from the NHIRD, provided by the NHIA, and the Ministry of Health and Welfare and was managed by National Health Research Institutes. The interpretation and conclusions contained herein do not represent those of the NHIA, Ministry of Health and Welfare, or National Health Research Institutes. The funding agencies had no role in study design, data collection and analyses, and result interpretation and publication of this study.

\section{Funding}

This study was supported in part by China Medical University under the Higher Education Sprout Project, Ministry of Education, Taiwan. It was also supported by the Taiwan Ministry of Health and Welfare Clinical Trial and Research Center of Excellence (MOHW107-TDU-B-212-123004), the Health and Welfare surcharge of tobacco product, China Medical University Hospital Cancer Research Center of Excellence (MOHW107-TDU-B-212-114-24) and Department of Medical Research, China Medical University Hospital (DMR107-004), Taiwan.

\section{Availability of data and materials}

All data of this study are deposited in a properly managed public repository. We examined and analysed datasets released from the Taiwan NHIRD (http://nhird.nhri.org.tw/en/index.html), maintained and managed by National Health Research Institutes (http://www.nhri.org.tw/), Taiwan. The use of the datasets is limited to research purposes only. Applicants must follow the Computer-Processed Personal Data Protection Law (http://www.winklerpartners.com/?p=987) and related regulations of National Health Insurance Administration and National Health Research Institutes, and the agreement must be signed by the applicant and his/her supervisor upon application submission. All applications are reviewed for approval of data release.

\section{Authors' contributions}

MYW conceived the study design, interpreted the data, and drafted the manuscript. $\mathrm{MCH}$ conceived the study design, and interpreted the data. $\mathrm{HHL}$ conceived the study design interpreted the data. JHC did the statistical analysis. YCL and CYH interpreted the data. MFS conceived the study design, interpreted the data, supervised the study, and finalized the manuscript. HRY conceived the study design, interpreted the data, supervised the study, and finalized the manuscript. MFS and HRY contributed equally. All authors read and approved the final manuscript.

\section{Ethics approval and consent to participate}

This study was approved by the Research Ethics Committee of China Medical University and Hospital, Taichung, Taiwan (CMUH104-REC2-115) and also the National Health Research Institute, the data holder of the NHI database. The patient consent was exempted for the total anonymity of all research data in this study.

\section{Consent for publication}

Not applicable in this section.

\section{Competing interests}

The funding agencies had no role in study design, data collection, analyses, result interpretation or publication of this study. This study was based in part on data from the NHIRD, provided by the NHIA, and the Ministry of Health and Welfare and was managed by National Health Research Institutes. The interpretation and conclusions contained herein do not represent those of the National Health Insurance Administration, Ministry of Health and Welfare, or National Health Research Institutes. All authors declare that they have no conflicts of interest.

\section{Publisher's Note}

Springer Nature remains neutral with regard to jurisdictional claims in published maps and institutional affiliations.

\section{Author details}

${ }^{1}$ Department of Chinese Medicine, China Medical University Hospital, 2 Yude Road, North District, Taichung 404, Taiwan. ${ }^{2}$ Graduate Institute of Chinese Medicine, School of Chinese Medicine, College of Chinese Medicine, China Medical University, 91 Hsueh-Shih Road, North District, Taichung 404, Taiwan. ${ }^{3}$ Dalin Tzu Chi Hospital, Buddhist Tzu Chi Medical Foundation, Chiayi 622, Taiwan. ${ }^{4}$ Management Office for Health Data, China Medical University Hospital, Taichung 404, Taiwan. ${ }^{5}$ School of Medicine, College of Medicine, China Medical University, Taichung 404, Taiwan. ${ }^{6}$ Graduate Institute of Acupuncture Science, College of Chinese Medicine, China Medical University, Taichung 404, Taiwan. ${ }^{7}$ Graduate Institute of Clinical Medical Science, China Medical University, Taichung 404, Taiwan. ${ }^{8}$ Research Center for Traditional Chinese Medicine, Department of Medical Research, China Medical University Hospital, 2 Yude Road, North District, Taichung 404, Taiwan. ${ }^{9}$ Research Center for Chinese Herbal Medicine, China Medical University, Taichung 404, Taiwan. ${ }^{10}$ Chinese Medicine Research Center, China Medical University, Taichung 404, Taiwan. ${ }^{11}$ Department of Biotechnology, Asia University, Taichung 413, Taiwan.

Received: 8 August 2018 Accepted: 26 November 2018

Published online: 22 December 2018

\section{References}

1. Firestein GS. Evolving concepts of rheumatoid arthritis. Nature. 2003; 423(6937):356-61.

2. Mclnnes IB, Schett $G$. The pathogenesis of rheumatoid arthritis. N Engl J Med. 2011;365(23):2205-19.

3. Smolen JS, Aletaha D, Mclnnes IB. Rheumatoid arthritis. Lancet. 2016; 388(10055):2023-38.

4. Nossent H. Risk of cardiovascular events and effect on mortality in patients with rheumatoid arthritis. J Rheumatol. 2000;27(9):2282-3.

5. Peters MJ, van Halm VP, Voskuyl AE, Smulders YM, Boers M, Lems WF, Visser M, Stehouwer CD, Dekker JM, Nijpels G, et al. Does rheumatoid arthritis equal diabetes mellitus as an independent risk factor for cardiovascular disease? A prospective study. Arthritis Rheum. 2009;61(11):1571-9.

6. Baghdadi LR, Woodman RJ, Shanahan EM, Mangoni AA. The impact of traditional cardiovascular risk factors on cardiovascular outcomes in patients with rheumatoid arthritis: a systematic review and meta-analysis. PLoS One. 2015;10(2):e0117952.

7. Hollan I, Dessein PH, Ronda N, Wasko MC, Svenungsson E, Agewall S, Cohen-Tervaert JW, Maki-Petaja K, Grundtvig M, Karpouzas GA, et al. Prevention of cardiovascular disease in rheumatoid arthritis. Autoimmun Rev. 2015;14(10):952-69.

8. De Vecchis R, Baldi C, Palmisani L. Protective effects of methotrexate against ischemic cardiovascular disorders in patients treated for rheumatoid arthritis or psoriasis: novel therapeutic insights coming from a meta-analysis of the literature data. Anatol J Cardiol. 2016;16(1):2-9.

9. Totoson P, Maguin-Gate K, Prigent-Tessier A, Monnier A, Verhoeven F, Marie C, Wendling D, Demougeot C. Etanercept improves endothelial function via pleiotropic effects in rat adjuvant-induced arthritis. Rheumatology (Oxford). 2016;55(7):1308-17.

10. Huang MC, Pai FT, Lin CC, Chang CM, Chang HH, Lee YC, Sun MF, Yen HR. Characteristics of traditional Chinese medicine use in patients with rheumatoid arthritis in Taiwan: a nationwide population-based study. J Ethnopharmacol. 2015;176:9-16. 
11. Liao HH, Yeh CC, Lin CC, Chen BC, Yeh MH, Chang KM, Sun MF, Yen HR. Prescription patterns of Chinese herbal products for patients with fractures in Taiwan: a nationwide population-based study. J Ethnopharmacol. 2015;173:11-9.

12. Torres-Rosas R, Yehia G, Pena G, Mishra P, Del Rocio Thompson-Bonilla M, Moreno-Eutimio MA, Arriaga-Pizano LA, Isibasi A, Ulloa L. dopamine mediates vagal modulation of the immune system by electroacupuncture. Nat Med. 2014;20(3):291-5

13. Ouyang BS, Gao J, Che JL, Zhang Y, Li J, Yang HZ, Hu TY, Yang M, Wu YJ, Ji LL. Effect of electro-acupuncture on tumor necrosis factor-alpha and vascular endothelial growth factor in peripheral blood and joint synovia of patients with rheumatoid arthritis. Chin J Integr Med. 2011;17(7):505-9.

14. Sato M, Inubushi M, Shiga T, Hirata K, Okamoto S, Kamibayashi T, Tanimura K, Tamaki N. Therapeutic effects of acupuncture in patients with rheumatoid arthritis: a prospective study using (18)F-FDG-PET. Ann Nucl Med. 2009;23(3):311-6.

15. Chang CC, Lee YC, Lin CC, Chang CH, Chiu CD, Chou LW, Sun MF, Yen HR. Characteristics of traditional Chinese medicine usage in patients with stroke in Taiwan: a nationwide population-based study. J Ethnopharmacol. 2016; 186:311-21.

16. NHIA. National Health Insurance Annu Rep 2015-2016. Taiwan: Taipie: National Health Insurance Administration, Ministry of Health and Welfare; 2015

17. Hsing AW, loannidis JP. Nationwide population science: lessons from the Taiwan National Health Insurance Research Database. JAMA Intern Med. 2015;175(9):1527-9.

18. Hung YM, Lin L, Chen CM, Chiou JY, Wang YH, Wang PY, Wei JC. The effect of anti-rheumatic medications for coronary artery diseases risk in patients with rheumatoid arthritis might be changed over time: a nationwide population-based cohort study. PLoS One. 2017;12(6):e0179081.

19. Chen YJ, Chang YT, Wang CB, Wu CY. The risk of cancer in patients with rheumatoid arthritis: a nationwide cohort study in Taiwan. Arthritis Rheum. 2011;63(2):352-8.

20. LaRosa JC, Pedersen TR, Somaratne R, Wasserman SM. Safety and effect of very low levels of low-density lipoprotein cholesterol on cardiovascular events. Am J Cardiol. 2013;111(8):1221-9.

21. Lee H, Lee JY, Kim YJ, Kim S, Yin C, Khil JH, Kwon K, Choi SM, Lee H, Park HJ. Acupuncture for symptom management of rheumatoid arthritis: a pilot study. Clin Rheumatol. 2008;27(5):641-5.

22. Zanette Sde A, Born IG, Brenol JC, Xavier RM. A pilot study of acupuncture as adjunctive treatment of rheumatoid arthritis. Clin Rheumatol. 2008;27(5): 627-35.

23. Steyers CM, 3rd, Miller FJ Jr.: Endothelial dysfunction in chronic inflammatory diseases. Int J Mol Sci 2014, 15(7):11324-11349.

24. Verhoeven F, Totoson P, Maguin-Gate K, Prigent-Tessier A, Marie C, Wendling D, Moretto J, Prati C, Demougeot C. Glucocorticoids improve endothelial function in rheumatoid arthritis: a study in rats with adjuvantinduced arthritis. Clin Exp Immunol. 2017;188(2):208-18.

25. Amaya-Amaya J, Sarmiento-Monroy JC, Mantilla RD, Pineda-Tamayo R, Rojas-Villarraga A, Anaya JM. Novel risk factors for cardiovascular disease in rheumatoid arthritis. Immunol Res. 2013;56(2-3):267-86.

26. Zhu J, Chen XY, Li LB, Yu XT, Zhou Y, Yang WJ, Liu Z, Zhao N, Fu C, Zhang $\mathrm{SH}$, et al. Electroacupuncture attenuates collagen-induced arthritis in rats through vasoactive intestinal peptide signallingdependent re-establishment of the regulatory $T$ cell/T-helper 17 cell balance. Acupunct Med. 2015;33(4):305-11.

27. Qi Y, Feng W, Song A, Song H, Yan S, Sun Q, Yang P. Role of serum IL-23/IL17 axis in the relationship between periodontitis and coronary heart disease. Int J Periodontics Restorative Dent. 2013;33(2):185-91.

28. Leem J. Acupuncture to treat hypertension: a recent systematic review and implications for subsequent research. Integr Med Res. 2016;5(1):69-71.

29. Firouzjaei A, Li GC, Wang N, Liu WX, Zhu BM. Comparative evaluation of the therapeutic effect of metformin monotherapy with metformin and acupuncture combined therapy on weight loss and insulin sensitivity in diabetic patients. Nutr Diabetes. 2016;6:e209.

30. Kim ST, Kim YJ, Lee JY, Lee H, Yin CS, Choi SM, Chae Y, Lee H, Park HJ. Acupuncture enhances superoxide dismutase (SOD) activity in the serum of rheumatoid arthritis patients. Clin Exp Rheumatol. 2009;27(2):384.

31. Bernateck M, Becker M, Schwake C, Hoy L, Passie T, Parlesak A, Fischer MJ, Fink M, Karst M. Adjuvant auricular electroacupuncture and autogenic training in rheumatoid arthritis: a randomized controlled trial. Auricular acupuncture and autogenic training in rheumatoid arthritis. Forsch Komplementmed. 2008;15(4):187-93.
32. Tam LS, Leung PC, Li TK, Zhang L, Li EK. Acupuncture in the treatment of rheumatoid arthritis: a double-blind controlled pilot study. BMC Complement Altern Med. 2007;7:35.

33. Wang $R$, Jiang $C$, Lei $Z$, Yin $K$. The role of different therapeutic courses in treating 47 cases of rheumatoid arthritis with acupuncture. J Tradit Chin Med. 2007;27(2):103-5.

34. David J, Townsend S, Sathanathan R, Kriss S, Dore CJ. The effect of acupuncture on patients with rheumatoid arthritis: a randomized, placebocontrolled cross-over study. Rheumatology (Oxford). 1999;38(9):864-9.

35. Man SC, Baragar FD. Preliminary clinical study of acupuncture in rheumatoid arthritis. J Rheumatol. 1974;1(1):126-9.

36. Sherman RE, Anderson SA, Dal Pan GJ, Gray GW, Gross T, Hunter NL, LaVange L, Marinac-Dabic D, Marks PW, Robb MA, et al. Real-world evidence - what is it and what can it tell us? N Engl J Med. 2016;375(23):2293-7.
Ready to submit your research? Choose BMC and benefit from:

- fast, convenient online submission

- thorough peer review by experienced researchers in your field

- rapid publication on acceptance

- support for research data, including large and complex data types

- gold Open Access which fosters wider collaboration and increased citations

- maximum visibility for your research: over $100 \mathrm{M}$ website views per year

At BMC, research is always in progress.

Learn more biomedcentral.com/submissions 